\title{
Adequacy Assessment of a Wind-Integrated System Using Neural Network-based Interval Predictions of Wind Power Generation and Load
}

\author{
Ronay Ak, Yan-Fu Li, Member, IEEE, Valeria Vitelli, and Enrico Zio, Senior Member, IEEE
}

\begin{abstract}
In this paper, we present a modeling and simulation framework for conducting the adequacy assessment of a windintegrated power system accounting for the associated uncertainties. A multi-perceptron artificial neural network (NN) is trained by a non-dominated sorting genetic algorithm-II (NSGA-II) to forecast point-values and prediction intervals (PIs) of the wind power and load. The output of the assessment is given in terms of point-valued and interval-valued Expected Energy Not Supplied (EENS). We consider different scenarios of wind power and load levels, to explore the influence of the uncertainty in wind and load predictions on the estimation of system adequacy.
\end{abstract}

Index Terms-Adequacy assessment, multi-objective geneticalgorithms, neural networks, prediction intervals.

\section{INTRODUCTION}

$\mathrm{T}$ HE ADEQUACY assessment of a power system is challenging due to the many uncertainties associated, for example, to fluctuations in energy demand, to the prediction of future weather conditions (e.g. wind speed, solar irradiation, etc.), to possible equipment (e.g. generators, lines, etc.) unavailability, to failures in electric power transactions, to errors (operator errors, dispatcher and relay malfunctions), and to other relevant issues [1]-[3].

In this paper, we present a modeling and simulation framework for conducting the adequacy assessment of a windintegrated power system accounting for uncertainties in the data and prediction models. A widely used adequacy index, the Expected Energy Not Supplied (EENS), is evaluated as output of the assessment. EENS measures the failure of the system to meet the demand by the cumulative amount of energy that is not provided to the customers, over the time horizon of interest for the analysis [4], [5].

Several works in the literature calculate EENS for the adequacy assessment of a power network [6]-[8]. The originality of the present work lies in proposing not only point-valued results, like the works previously mentioned, but also interval-valued results to inform the decision makers on the uncertainty in the predictions. Uncertainties are here considered due to load fluctuations, wind variability, and component failures.

A case study is considered in which hourly wind speed data from the region of Regina, Saskatchewan, Canada are taken, from a 9-year period (1 Jan. 2003 to 31 Dec. 2011) [9]. Hourly mean wind speed data are used to determine the timedependent wind power output of a wind turbine generator (WTG) using its power curve [7]. For load demand, the hourly load fluctuations are modeled using the chronological annual load curve of the IEEE Reliability Test System (RTS) [10] with the scaled annual peak load value. The inputs to estimate the EENS are the Prediction Interval (PIs) for 1-hour ahead wind power and load. These values are provided by the use of a multi- perceptron artificial neural networks (NNs) trained by the non-dominated sorting genetic algorithm-II (NSGA-II) [11]: the lower and upper bounds of the NN-based PIs are optimal both in terms of coverage probability (PICP) and of width (PIW). The NSGA-II training procedure generates Pareto-optimal solution sets, which include non-dominated solutions for the two objectives (PICP and PIW). One solution has, then, to be selected among the ones in the Pareto optimal set according to the preferences on the objectives.

The generating units in the power system are represented by two-state models, describing operation and failure, and they are sampled by sequential Monte Carlo simulation.

\section{Methodology to Estimate LoAD And Wind Power PIs}

In the following sub-sections, the main phases of the methodology are described. The application of the framework is shown on a case study taken from literature [7]. In Fig. 1, a flowchart of the methodology for the adequacy assessment of wind-integrated power systems is depicted.

\section{A. Wind Power Generation}

Hourly wind speed data have been collected for the region of Regina, Saskatchewan, Canada for a 9-year period (1 Jan. 2003 to 31 Dec. 2011) [9]. Since wind power is a function of wind speed, forecasts of power are generally derived from wind speed. In order to conduct the adequacy assessment over one-year time horizon, for each hour in the year $(8736 \mathrm{~h})$ the hourly means are calculated over 9 years of wind speed values. The so obtained one-year time series of wind speed $V(t), t=1, \ldots, 8736$, are then transformed in wind power $P(t)$ values using a quadratic characteristic curve (power curve) of 
literature [12], [13]. In the numerical case study, the parameters defining the power curve, i.e. cut-in speed, rated speed, cut-off speed and rated capacity, are set to $14.4 \mathrm{~km} / \mathrm{h}$, $36 \mathrm{~km} / \mathrm{h}, 80 \mathrm{~km} / \mathrm{h}$ and $40 \mathrm{MW}$, respectively [7].

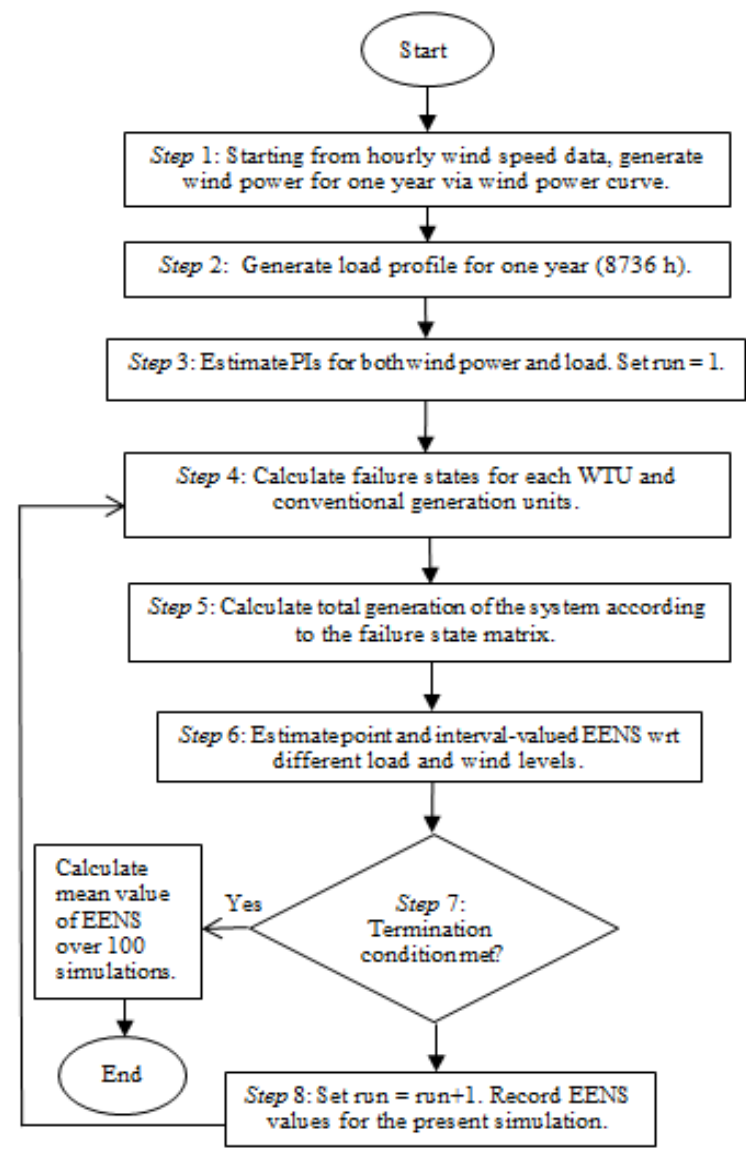

Fig. 1. Flowchart of the proposed methodology.

\section{B. Load Modeling}

The load duration curve (LDC) on an annual basis (8736 h) is created by manipulating the hourly load values from the IEEE-RTS [10]. One year ( $8736 \mathrm{~h})$ load data, i.e. a load value $L(t)$ for each hour $t=1, \ldots, 8736$, have been generated with the following formula [14]:

$$
L(t)=\bar{L}(t)+\bar{L}(t)\left(\frac{\sigma}{100}\right) X^{n o r m}
$$

where $\bar{L}(t)$ is the expected value of load for hour $t$, calculated using the following equation:

$$
\bar{L}(t)=P_{w}(t) \times P_{d}(t) \times P_{h}(t) \times L_{\text {max }}
$$

where $L_{\text {max }}$ is the peak load in a year, $P_{w}(t)$ is the weekly peak load as a percentage of the annual peak, $P_{d}(t)$ is the daily peak load as a percentage of the weekly peak and $P_{h}(t)$ is the hourly peak load as a percentage of the daily peak. The system peak load $L_{\max }$ is set to $185 \mathrm{MW}$ [7]. $\sigma$ is the load forecasting uncertainty error (standard deviation) expressed as a percentage of the hourly peak load, and $X^{\text {norm }}$ is defined as [14]:

$$
X^{\text {norm }}=\sqrt{-2 \ln \left(R_{1}\right)} \cos \left(2 \pi R_{2}\right)
$$

where $R_{1}$ and $R_{2}$ are two random numbers drawn from the standard uniform distribution on the open interval $(0,1)$. The load forecasting error $\sigma$ is set to $5 \%$.

\section{Estimation of NN-based PIs}

Based on the hourly wind power and load values over a 1year horizon, we define a data-driven strategy to perform short term (1-hour ahead) prediction, with uncertainty quantification, of both load and wind power. Not only a point estimate of the target, but also PIs are computed. In order to estimate PIs for 1-hour ahead wind power and load prediction, we use multi-perceptron artificial neural networks (NNs) [15], [16] which are a class of nonlinear statistical models inspired by brain architecture. NNs are capable of learning complex nonlinear relationships among variables from observed data by a process of parameter tuning called "training" [16] - [18].

A PI is comprised of upper and lower bounds in which a future unknown value of the target is expected to lie with a predetermined confidence level $(1-\propto)$ [18]. We evaluate the "goodness" of the PIs by estimating the empirical PIs coverage probability (PICP), which one wants to maximize, and the interval width (PIW), which one wants to minimize. The mathematical definition of the PICP and PIW used in this work is defined in [18], [19].

The PIs estimation problem is addressed by taking into account these two conflicting objectives within a multiobjective framework. For this, we use NSGA-II, one of the most efficient multi-objective genetic algorithms (MOGAs) [11], [20] to optimize the parameters (i.e. the weights $\widehat{w}$ ) of the NN with respect to both PICP and PIW objectives. More precisely, the NN is trained by NSGA-II to produce the lower and upper bounds of the PIs for short-term forecasting (1-hour ahead) of wind power and load. For the details of the practical implementation of NSGA-II for NN-based PIs estimation see [19].

\section{Methodology to Estimate EENS}

In order to conduct the adequacy assessment of the windintegrated power system, we use the well-known adequacy index, EENS, which quantifies the capability of the system to meet the demand in the time horizon considered for the analysis. EENS measures the expected value of the energy not supplied due to the lack of available energy through the given time horizon (e.g. one year). It depends on the predicted values for both the system energy production and the power demand, and it is formulated as follows [21], [22]:

$$
E E N S=\sum_{t=1}^{N} \operatorname{Pr}\left(L_{t}>G_{t}\right) \times\left(L_{t}-G_{t}\right)
$$

where $t$ is the equally sized time step (e.g. hour or day), $N$ is 
the total number of time steps in the considered time horizon, in our case $N=8736$ for a one year time horizon, $G_{t}$ is the total power generation available at time step $t, L_{t}$ is the load demand at time step $t, \operatorname{Pr}\left(L_{t}>G_{t}\right)$ is the probability that the load demand exceeds the available power generation at time step $t$.

In the classical definition of EENS given in (4), both the predicted value of the generation $G_{t}$ and of the load $L_{t}$ at each time step $t$ are assumed to be point estimates, resulting in a point estimate of EENS. Our method is, instead, capable of providing PIs for both the power generation and the load at each time step, to take into account the possible uncertainties in the prediction arising from both the underlying physical processes (wind inherent uncertainty, variability in power demand, ...) and in the system stochastic behavior (equipment failures, approximations of the system complexities, ...). A proper adequacy assessment model should take these sources of uncertainty into account, since uncertainty quantification is crucial for a real understanding of the system behavior, and for obtaining reliable results useful for robust decision making. Hence, we aim at a generalization of the EENS formulation given in (4), in order to include interval estimates of both $G_{t}$ and $L_{t}$.

Two different strategies are considered for interval-based EENS estimation: a point estimation and an interval estimation. They are both interval-based, in the sense that the inputs to the evaluation are the short-term PIs for load and for power generation, as obtained by the NN-based estimation procedure described in the previous section.

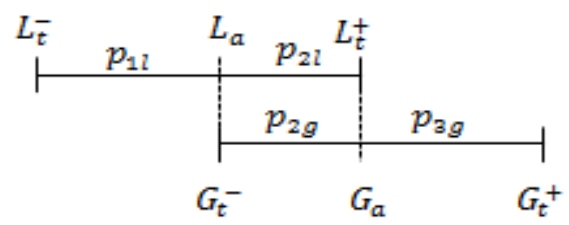

(a)

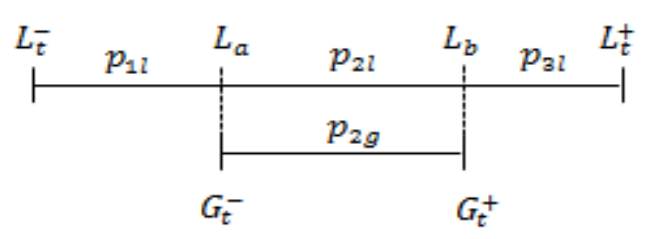

(b)

Fig. 2. Two different cases describing possible load and generation at time $t$.

\section{A. Interval-based Interval Estimation of EENSs}

One possible strategy for taking into account load and power generation PIs in EENS estimation consists in directly using (4) with interval-valued $G_{t}$ and $L_{t}$, thus obtaining as a result an interval evaluation of EENS by directly applying the principles of interval arithmetic [23]. In other words, all arithmetic calculations throughout the evaluation process of the interval-valued $G_{t}$ and $L_{t}$ are performed according to interval arithmetic (interval product, sum, intersection, etc.). Moreover, an assumption is made in the computation of $\operatorname{Pr}\left(L_{t}>G_{t}\right)$ in the case of interval- valued $L_{t}$ and $G_{\mathrm{t}}$ : due to lack of further information, a uniform probability is assumed for the actual (unknown) values of both load and power generation being anywhere inside the intervals of $L_{t}$ and $G_{t}$, respectively.

This way of proceeding seems the most natural for the generalization of the classical EENS index to take into account interval estimates of load and power generation. In fact, in this way the uncertainty quantification attained in the load and power PIs estimation process is directly propagated into the adequacy assessment index, without any strong probabilistic assumption. Moreover, since the EENS estimate is based on uncertain quantities, it is uncertain itself, and thus it is natural to quantify its uncertainty via an interval. Finally, an interval EENS carries richer information which can eventually be converted in a single value whenever needed.

More specifically, total load and total generation at time $t$ are defined as $L_{t}=\left[L_{t}^{-}, L_{t}^{+}\right]$and $G_{t}=\left[G_{t}^{-}, G_{t}^{+}\right]$, respectively, where $L_{t}^{-}$and $G_{t}^{-}$indicate the lower bounds, and $L_{t}^{+}$and $G_{t}^{+}$ indicate the upper bounds of the intervals of the two quantities.

$\left(L_{t}-G_{t}\right)$ in (4) is calculated as follows, in accordance with the interval arithmetic rules [23]:

$$
\left(L_{t}-G_{t}\right)=\left[L_{t}^{-}-G_{t}^{+}, L_{t}^{+}-G_{t}^{-}\right]
$$

After subtraction, if the lower bound of the interval becomes a negative value, it is replaced by 0 .

For the computation of $\operatorname{Pr}\left(L_{t}>G_{t}\right)$, different cases defined by load and generation interval-valued levels at time step $t$ have to be considered. For the sake of brevity, two examples are considered for exemplification, and shown in Fig. 2.

For the case in Fig. 2(a), $\operatorname{Pr}\left(L_{t}>G_{t}\right)$ is calculated as follows:

$$
\operatorname{Pr}\left(L_{t}>G_{t}\right)=p_{2 l} \times p_{2 g} \times 1 / 2
$$

where $p_{1 l}, p_{2 l}$, and $p_{2 g}, \mathrm{p}_{3 \mathrm{~g}}$ are fractions of the intervals $L_{t}$ and $G_{t}$, respectively. Specifically, if $\operatorname{diam}()$ indicates the length of an interval, $p_{1 l}$ is the fraction of $\operatorname{diam}\left(L_{t}^{-}, L_{a}\right)$ over the length of the entire interval, $\operatorname{diam}\left(L_{t}^{-}, L_{t}^{+}\right)$. This fraction corresponds to the probability of the actual (unknown) load (or generation) being within that part (subinterval) of the interval, because of the assumption of uniform distribution of the actual load (or generation) within the estimated intervals. In fact, we can formally derive (6) by directly using the probability density function of a uniform random variable [24]: if $U$ is a uniform random variable on the interval $(m, n)$, then its probability density function $\phi(u)$ is given by

$$
\phi(u)=\left\{\begin{array}{cc}
\frac{1}{m-n}, & \text { if } m<u<n \\
0, & \text { otherwise }
\end{array}\right.
$$


If $[a, b]$ is a subinterval of $(m, n)$, then the probability of $U$ falling within the interval $[a, b]$ depends only on the length of $[a, b]$ with respect to $(m, n)$. Specifically [24]:

$$
\operatorname{Pr}(a \leq U \leq b)=\int_{a}^{b} \frac{d u}{m-n}=\frac{a-b}{m-n}
$$

If the load and the generation intervals are as in Fig. 2(b), $\operatorname{Pr}\left(L_{t}>G_{t}\right)$ is calculated similarly as follows:

$$
\operatorname{Pr}\left(L_{t}>G_{t}\right)=p_{3 l}+p_{2 l} \times p_{2 g} \times 1 / 2
$$

where $p_{3 l}, p_{2 l}$, and $p_{2 g}$ are the intervals fractions as defined above. This calculation corresponds to the probabilistic assumption that load and generation can take any value in $\left[L_{t}^{-}, L_{t}^{+}\right]$and $\left[G_{t}^{-}, G_{t}^{+}\right]$, respectively, with uniform probability, i.e. each point in $\left[L_{t}^{-}, L_{t}^{+}\right]$and $\left[G_{t}^{-}, G_{t}^{+}\right]$is equally likely to be a possible value of $L$ and $G$, respectively.

\section{B. Interval-based Point Estimation of EENSs}

As explained in Section 3.A, load and power generation, provided by NNs as PIs, can be directly used for EENS estimation. One possible strategy, leading to an interval estimation of EENS, has already been described in the previous section. An alternative way to generalize EENS to the interval case leads to obtaining a point estimate of the adequacy index. This strategy is based on the probability density function of the continuous random variable $\xi_{t}=l_{t}-$ $g_{t}$, where $l_{t} \in L_{t}$ and $g_{t} \in G_{t}$ are, respectively, two admissible values of the load demand and power generation at time $t$, thus $\xi_{t} \in\left[\max \left\{0, L_{t}^{-}-G_{t}^{+}\right\}, L_{t}^{+}-G_{t}^{-}\right]$. Any value assumed by $\xi_{t}$ represents a possible amount of energy that cannot be supplied by the power system at time $t$ to meet the demand: hence, a point estimate of EENS at time $t$ can be obtained by computing the expected value of $\xi_{t}$ over the intervals of admissible values for load and power, $L_{t}$ and $G_{t}$, respectively. This is indeed a probabilistic approach, since the assumption of uniform distribution of the energy values within $L_{t}$ and $G_{t}$ has again to be made. Moreover, uncertainty quantification is taken into account, because the load and power PIs are used in the EENS estimation process. The obtained final estimate of EENS is a single value, which may give a more interpretable result.

According to this strategy, the EENS of the system can be calculated as follows [22]:

$$
E E N S=\int_{\max \left\{0, L_{t}^{-}-G_{t}^{+}\right\}}^{L_{t}^{+}-G_{t}^{-}} \xi_{t} P\left(\xi_{t}>0\right) d \xi_{t}
$$

From this general formulation we can derive the following expressions, for the examples shown in Fig. 2 (Fig. 2a and 2b, respectively):

$$
E E N S=\int_{G_{t}^{-}}^{L_{t}^{+}} d L_{t} \int_{G_{t}^{-}}^{L_{t}}\left(L_{t}-G_{t}\right) \frac{1}{W_{L}} \frac{1}{W_{G}} d G_{t}
$$

$$
E E N S=\int_{G_{t}^{-}}^{G_{t}^{+}} d G_{t} \int_{G_{t}}^{L_{i}^{+}}\left(L_{t}-G_{t}\right) \frac{1}{W_{L}} \frac{1}{W_{G}} d L_{t}
$$

where $W_{L}=L_{i}^{+}-L_{t}^{-}$and $W_{G}=G_{t}^{+}-G_{t}^{-}$, and we directly computed the integrals assuming a uniform probability density function for both random variables $L_{t}$ and $G_{t}$. In general, for any of the possible cases of interval-valued load and generation at each time step, we can derive an analytic expression for the interval-based point estimate of EENS. We do not report the explicit EENS calculations in each case, for the sake of brevity.

\section{EXPERIMENTAL RESULTS}

The proposed approach has been tested on the RBTS (Roy Billinton test system) system [25]. The RBTS system consists of 11 conventional generation units with a total capacity of $240 \mathrm{MW}$. A wind farm with 20 identical WTG units has been added to the RBTS system. Each WTG is assumed to have a rated capacity of $2 \mathrm{MW}$ and cut-in, rated and cut-out speeds of $14.4 \mathrm{~km} / \mathrm{h}, 36 \mathrm{~km} / \mathrm{h}$ and $80 \mathrm{~km} / \mathrm{h}$, respectively. In Fig. 3, the system topology of the RBTS system is shown.

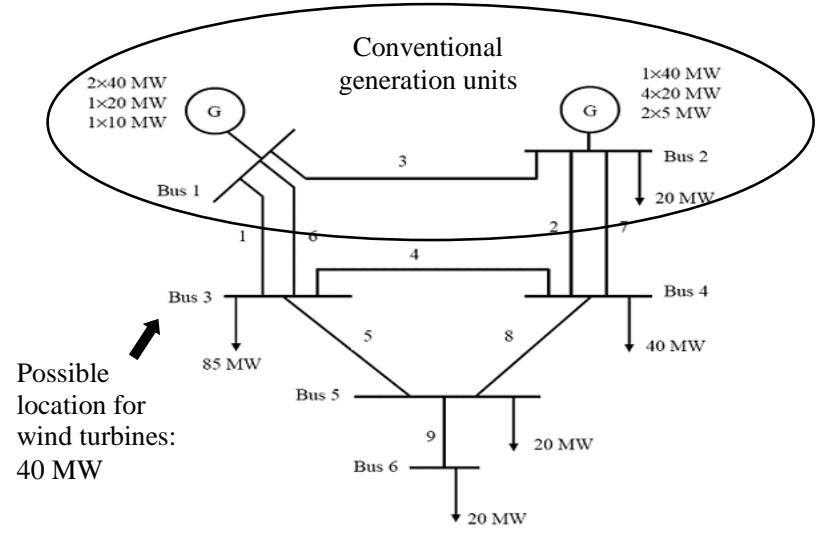

Fig. 3. Single line diagram of the RBTS [7].

\section{A. Failure Modeling}

With respect to the failure and repair behavior, the system components are considered to be independent and with only two states: up and down.

It is assumed that all components are initially in the up state. For a generic component $i$ (such as generator, transformer, line, etc.), both time-to-failure $\left(\mathrm{TTF}_{i}\right)$ and timeto-repair $\left(\mathrm{TTR}_{i}\right)$ follow an exponential distribution. By randomly sampling two numbers $R_{1}$ and $R_{2}$ from a uniform distribution, the sampled values of the state residence time are calculated as follows:

$$
\begin{gathered}
T T F=-M T T F \times \ln \left(R_{1}\right) \\
T T R=-M T T R \times \ln \left(R_{2}\right)
\end{gathered}
$$

where MTTF and MTTR are the means of the respective exponential distributions. 
Table I reports conventional generating unit ratings and reliability data [25]. For an individual wind turbine, the failure and repair rates are set to $0.0005 / \mathrm{hr}$ and $0.013 / \mathrm{hr}$, respectively [2].

TABLE I

CONVENTIONAL GENERATION UNITS' RELIABILITY DATA [25]

\begin{tabular}{ccccc}
\hline $\begin{array}{c}\text { Unit size } \\
\text { (MW) }\end{array}$ & Type & $\begin{array}{c}\text { No. of } \\
\text { units }\end{array}$ & $\begin{array}{c}\text { MTTF } \\
\text { (hr) }\end{array}$ & $\begin{array}{c}\text { MTTR } \\
\text { (hr) }\end{array}$ \\
\hline 5 & hydro & 2 & 4380 & 45 \\
10 & thermal & 1 & 2190 & 45 \\
20 & hydro & 4 & 3650 & 55 \\
20 & thermal & 1 & 1752 & 45 \\
40 & hydro & 1 & 2920 & 60 \\
40 & thermal & 2 & 1460 & 45 \\
\hline
\end{tabular}

\section{B. Data Description and NN Parameters}

Hourly wind speed time data for the period 2003-2011 (9 year series) have been measured in Regina, Saskatchewan, a region of central Canada [9]. These 9 years data have been used to calculate hourly mean wind speed values. The one year time series of wind speed have then been transformed in a time series of wind power through the characteristic curve (power curve) of a wind turbine, defined in Section 2.A. One year $(8736 \mathrm{~h})$ load data, i.e. load profile over 1 year with 1 -h time step, have been generated according to the load model described in Section 2.B. Fig. 4 shows raw time series data sets, for both total wind power of WTG units, with a maximum value of $37.36 \mathrm{MW}$ and load, with a maximum value of $196.88 \mathrm{MW}$. Both time series data sets show remarkable fluctuations along time.

The architecture of the NN model used consists of one input, one hidden and one output layers. The number of input neurons is set to 4 for both load and wind PIs estimations, since an auto-correlation analysis [26] has shown that the historical past values $x_{t-1}, x_{t-2}, x_{t-3}$, and $x_{t-4}$ should be used as input variables for predicting $x_{t}$ in output; the number of hidden neurons is set to 10 after a trial-and-error process; the number of output neurons is set to 2 , to provide the lower and upper bounds. As activation functions, the hyperbolic tangent function in the hidden layer and the logarithmic sigmoid function in the output layer have been found to give the most satisfactory results. In the NSGA-II, population size is set to 50 and the number of generations to 300 . To account for the inherent randomness of NSGA-II, five different runs have been performed and an overall best nondominated Pareto front has been obtained from the five individual fronts. All data have been normalized within the range [0.1, 0.9].

\section{Estimated PIs}

The multi-objective NSGA-II with PI coverage probability and width provide Pareto sets of solutions (one for the wind power and one for the load), i.e. optimal NN models (weights); it is, then, necessary to select the optimal sets of weights to use in the NN models for prediction (see Fig. 5). For exemplification purposes, solutions are here subjectively chosen as a good compromise in terms of high PICP and low NMPIW. The selected solutions are characterized by $95 \%$ PICP and a NMPIW equal to 0.265 for the load prediction, and $95 \%$ PICP with a NMPIW equal to 0.19 for the wind power prediction, respectively. Fig. 6 shows 1-hour ahead PIs for the selected Pareto solutions, marked in rectangles in Fig. 5 , estimated by the trained NNs for wind power from one turbine and load predictions. For the sake of clarity of visualization, a zoom on the first 250 hours has been plotted.

TABLE II

DESCRIPTIVE STATISTICS OF EENS OVER 100 SIMULATIONS

\begin{tabular}{ccc}
\hline & Mean & Std dev. \\
\hline EENS_LB & 19278.93 & 11090.22 \\
EENS_UB & 25521.84 & 14663.75 \\
EENS_mean & 22381.89 & 12859.20 \\
EENS_point & 22419.12 & 12888.83 \\
EENS_actual & 22769.24 & 13147.55 \\
\hline
\end{tabular}

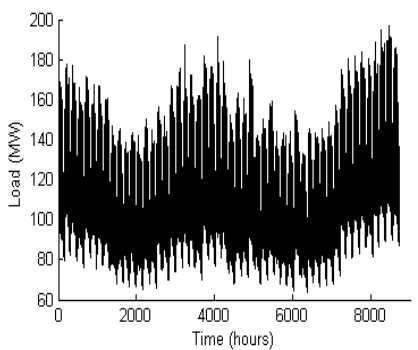

(a)

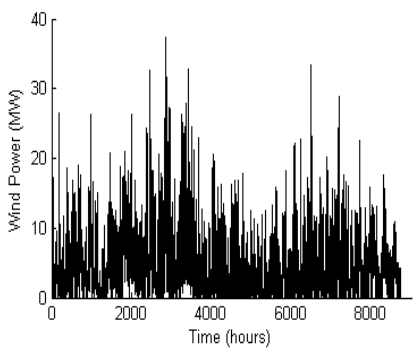

(b)
Fig. 4. The wind power time series set and load curve over 1 year used in this study: (a) load (b) wind power.

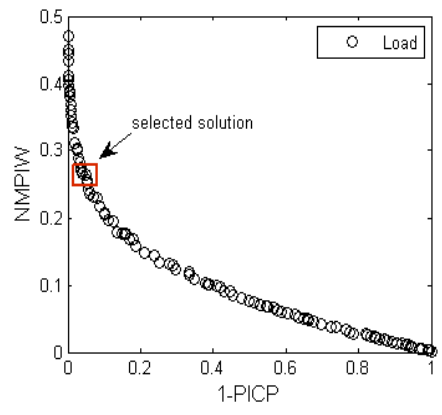

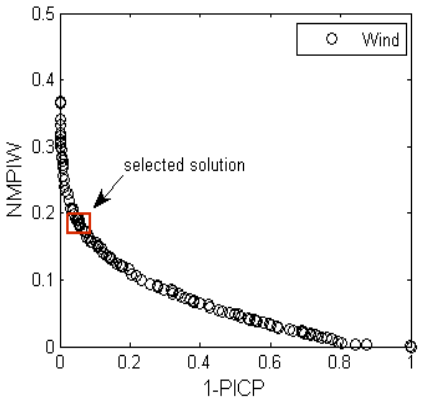

Fig. 5. The overall best Pareto front obtained by training of the NNs for $1 \mathrm{~h}$ ahead load and wind power predictions: (a) load (b) wind power.

\section{Estimated EENS}

To estimate the overall EENS accounting for failures and repairs of the components, we performed 100 repetitions. In each repetition, a new matrix of the up and down states of the components is generated. Then, for each repetition the assessment process is followed with the same estimated load 
and wind power PIs and conventional units' generation capacity. Fig. 7 shows the EENS results obtained according to the methods explained in Sections 3.A and 3.B. It can be noticed that the estimated PIs of EENS include the point predictions. In other words, interval-valued EENS carries more information, i.e. reflects the worst and best cases of unavailable energy during the given time horizon, and it provides an indication of how the uncertainties in input affect the output quantities.

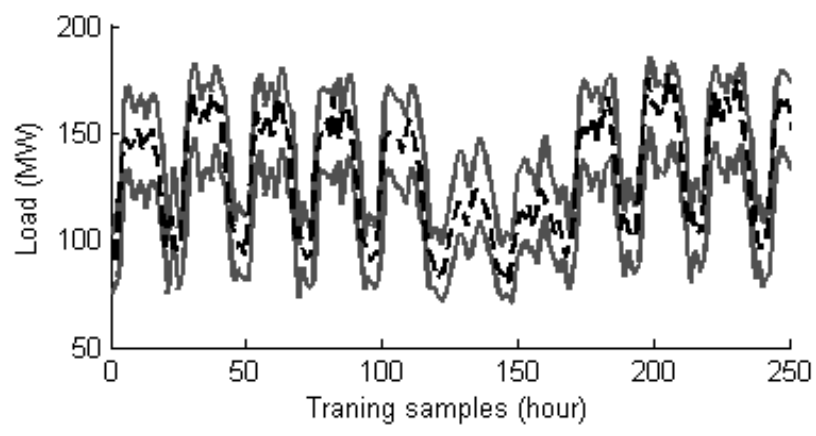

(a)

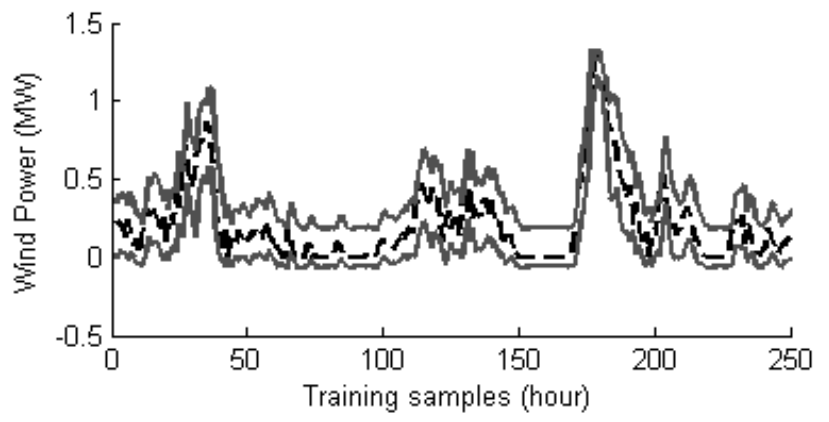

(b)

Fig. 6. Estimated PIs (solid lines) over a 1-year time horizon (dashed line): (a) $1 \mathrm{~h}$-ahead load (b) $1 \mathrm{~h}$-ahead wind power from one turbine.

The EENS values in Fig. 7 (b) have been obtained by considering 6 different scenarios, corresponding to the different uncertainty levels in the input parameters, i.e. wind power, load and system state. These scenarios have been called point EENS, interval EENS, EENS LB, EENS mean, EENS UB and EENS actual. The former two are the same as in Fig. 7 (a). EENS LB and EENS UB have been calculated by considering only the LB and UB of the estimated load and wind power PIs, respectively, and by computing a singlevalued inputs EENS index. Similarly, to estimate EENS mean, the central values (mean point) of the PIs have been used as input. For computing EENS actual, we have used the actual data sets shown in Fig. 4: EENS actual is, thus, the unknown quantity we would like our estimates to be close to, and it cannot be computed in a real case study; we have calculated it here only for demonstration of the strength of our approach. Note that, differently from the point EENS and interval EENS, the values of EENS LB, EENS UB, and EENS mean are calculated with single-valued load and wind power inputs: hence, they do not include any uncertainty quantification in their estimation process. Table II reports the mean and standard deviations of the EENS LB, EENS UB, EENS mean,
EENS point and EENS actual results over 100 simulations. Note that, being capable of properly accounting also for uncertainties, EENS point is the closest to EENS actual on average (and with comparable variability).

EENS is equal to zero when there is no failure of conventional generators (see Fig. 7), because at any time $t$ over the given time horizon, total available generation capacity, $G(t)$ is bigger than total load $L(t)$. In case of failures, EENS takes different non-zero values according to the load and wind levels. Standard deviations show the effect of the failures on the system adequacy, since load and wind levels do not change through the runs. One can, then, use the results of such analysis to identify the components which have high contribution to the expected annual energy not supplied. Also, if the estimated EENS is unacceptable, technical interventions may be needed, e.g. new generation units might be added to the system.

As each scenario carries different information, ultimately the decision makers are supposed to select the one which gives a more interpretable result for their final decisions/actions. Fig. 8 shows the boxplots of the differences obtained by the subtraction of ENNS_actual from the EENS_LB, EENS_UB, EENS_mean and EENS_point, respectively. A boxplot is an exploratory graphic used to visualize key statistical measures, such as median and quartiles, and to have an idea about the distribution of a data set, i.e. the location, dispersion, and symmetry or skewness of the data set, at a glance [27], [28]. It is also used to make comparisons of these features in two or more data sets. The boxplots dif_mean and dif_point are comparatively shorter (meaning narrower distributions) than the boxplots dif_LB and dif_UB. This fact indicates a higher variability for the estimates of EENS obtained using PIs LB and UB, compared to the ones based on EENS point and mean. In other words, EENS mean and EENS point show comparable results, which are also more consistent with respect to the actual values of EENS throughout the simulations (not just on average, as we could already conclude from Table II). Since the EENS point is estimated on the basis of the load and power PIs, i.e. it takes into account the uncertainties in the inputs, it is more precise and reliable compared to the others. Hence, among all the possible estimates of EENS that could be obtained, EENS point shows more promising and trustable results in capturing the actual EENS by considering the uncertain inputs.

On the basis of the comparisons shown in Fig. 7 and Table II, where load demand and wind power generation take different values according to the considered scenario, the conclusion that different load and wind levels result in different EENS can be drawn. From the results reported in Table II we can observe that, for values of the load corresponding to the upper bound of PIs, a bigger EENS is obtained compared to the one obtained in other scenarios. It is worth to remark that, in the same scenario, an increase in the wind level would reduce the EENS. Ultimately, having an estimate of EENS with an associated variability helps the decision makers in managing the system on the basis of a more realistic / reliable adequacy assessment. 


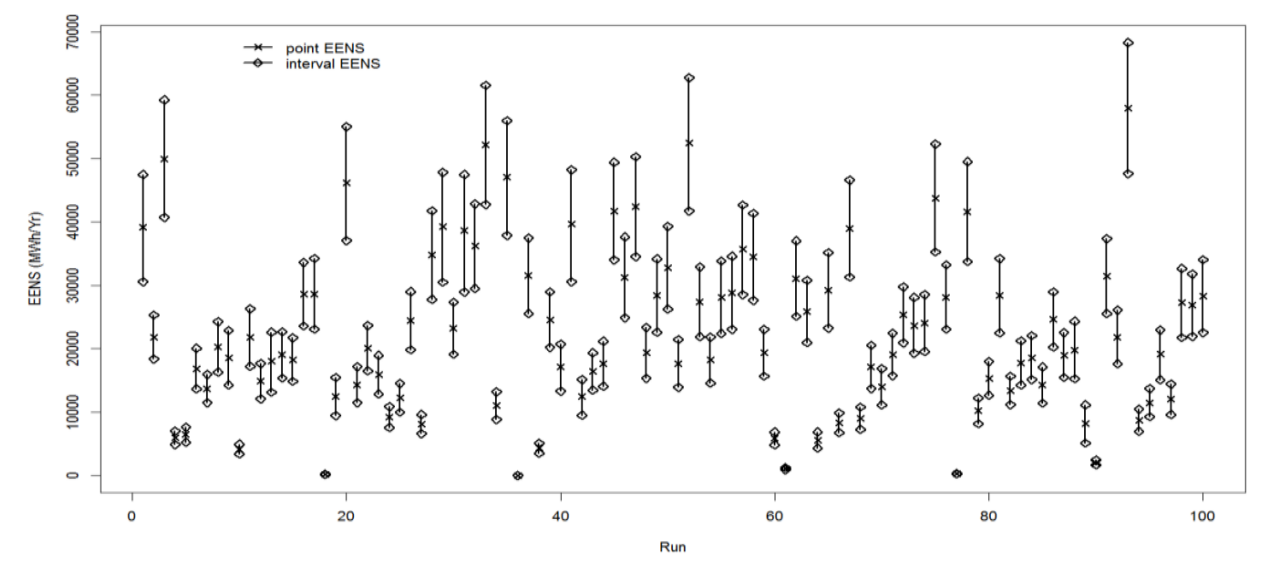

(a)

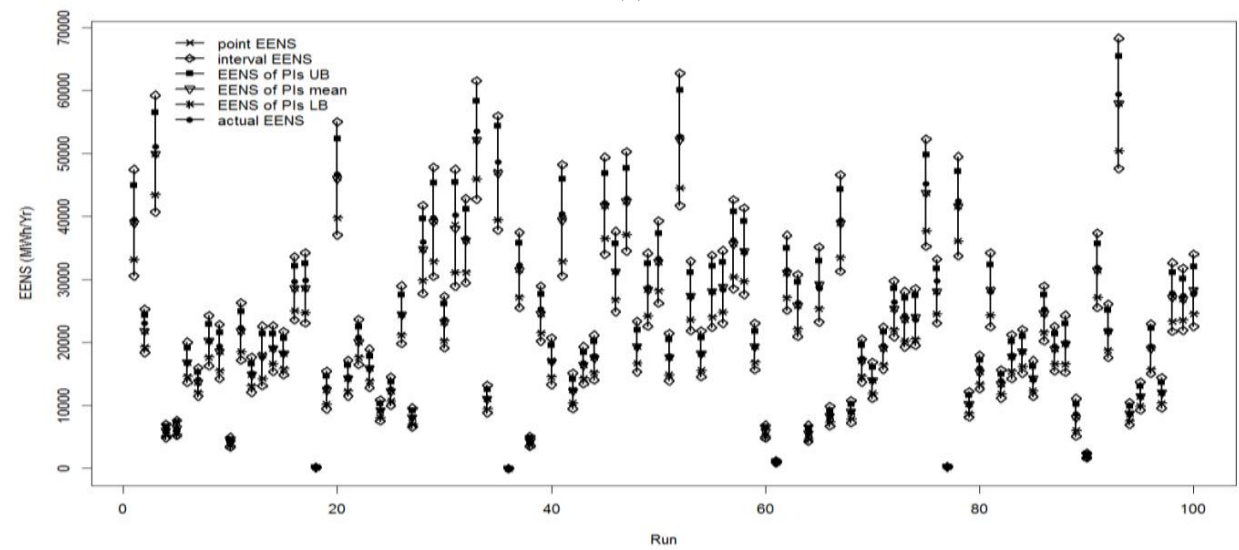

(b)

Fig. 7. The EENS results over 100 runs: (a) interval-valued and single-valued EENS (b) comparisons of different scenarios.

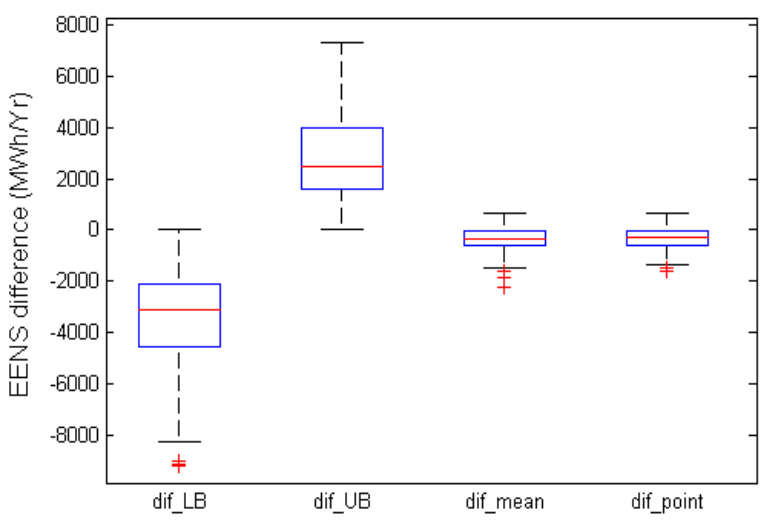

Fig. 8. Boxplots of the differences obtained by the subtraction of ENNS_actual from the EENS_LB, EENS_UB, EENS_mean and EENS_point, respectively.

\section{CONCLUSION}

A method which calculates the EENS value for a windintegrated power network based on interval-valued load and wind power input data has been proposed. The objective is to know and dominate the impact of the uncertainty in wind and load on the uncertainty in EENS. Simulation results on different scenarios confirm that uncertainties in input data can be properly taken into account to obtain more reliable EENS estimations.

The presented expected annual energy not supplied can be integrated with a cost model whose results help the decision makers to take operational level decisions and do mediumterm and long-term strategic planning.

\section{REFERENCES}

[1] E. Zio and T. Aven, "Uncertainties in smart grids behavior and modeling: What are the risks and vulnerabilities? How to analyze them?," Energy Policy, vol. 39, no. 10, pp. 6308-6320, Oct. 2011.

[2] Y-F. Li and E. Zio, "A multi-state model for the reliability assessment of a distributed generation system via universal generating function," Reliability Engineering \& System Safety, vol. 106, pp. 28-36, Oct. 2012.

[3] Z. Wei, T. Tao, D. ZhuoShu, and E. Zio, "A dynamic particle filtersupport vector regression method for reliability prediction," Reliability Engineering \& System Safety, vol. 119, pp. 109-116, Nov. 2013.

[4] R., Billinton and R. N. Allan, Reliability Evaluation of Power Systems. 2nd ed., New York: Plenum Press, 1996.

[5] G. A. Koeppel, "Reliability considerations of future energy systems: multi-carrier systems and the effect of energy storage," Ph.D. dissertation, Power System Laboratory, Swiss Federal Institute of Technology, Zurich, 2007. 
[6] J. Wen, Y. Zheng, and F. Donghan, "A review on reliability assessment for wind power," Renewable and Sustainable Energy Reviews, vol. 13, no. 9, pp. 2485-2494, Dec. 2009.

[7] Y. Gao, R. Billinton, and R. Karki, "Composite generation and transmission system adequacy assessment considering wind energy seasonal characteristics," presented at the IEEE Power Energy Society General Meeting, PES '09 IEEE, Calgary, Canada, July 26-30, 2009.

[8] B. Falahati, Y. Fu, Z. Darabi, and L. Wu, "Reliability assessment of power systems considering the large-scale PHEV integration," presented at the Vehicle Power and Propulsion Conference (VPPC), 2011 IEEE, Chicago, USA, Sep. 6-9, 2011.

[9] Canadian Weather Office. [Online]. Available: http: //www.weatheroffice.gc.ca/canada_e.html. [Accessed: 01-Jan-2013].

[10] Reliability Test System Task Force of the Application of Probability Methods Subcommittee, "IEEE Reliability Test System," IEEE Trans. on Power Apparatus and Systems, vol. PAS-98, no. 6, pp. 2047-2054, Nov. 1979.

[11] K. Deb, A. Pratap, S. Agarwal, and T. Meyarivan, "A fast and elitist multiobjective genetic algorithm: NSGA-II," IEEE Transactions on Evolutionary Computation, vol. 6, no. 2, pp. 182-197, Apr. 2002.

[12] R. Karki, P. Hu, and R. Billinton, "A simplified wind power generation model for reliability evaluation," IEEE Trans. on Energy Conversion, vol. 21 , no. 2 , pp. 533-540, June 2006

[13] C.G. Justus, W.R. Hargraves, and A. Yalcin, "Nationwide assessment of potential output from wind-powered generators," Journal of Applied Meteorology, vol. 15, no.7, pp. 673-678, July 1976.

[14] A.B. Rodrigues and M.G. Da Silva, "Probabilistic assessment of available transfer capability based on Monte Carlo method with sequential simulation," IEEE Transactions on Power Systems, vol. 22, no. 1, pp. 484-492, Feb. 2007

[15] J., Korbicz, J. M. Ko'scielny, Z. Kowalczuk, and W. Cholewa, Fault Diagnosis: Models, Artificial Intelligence, Applications. Germany: Springer Verlag, 2004.

[16] K. Hornik, M. Stinchcombe, and H. White, "Multilayer feedforward networks are universal approximators," Neural Networks, vol. 2, no. 5, pp. 359-366, 1989.

[17] D.L. Shrestha and D.P. Solomatine, "Machine learning approaches for estimation of prediction interval for the model output," Neural Networks vol. 19, no. 2, pp. 225-235, 2006.

[18] A. Khosravi, S. Nahavandi, D. Creighton, and A. F. Atiya, "Lower Upper Bound Estimation Method for Construction of Neural NetworkBased Prediction Intervals," IEEE Trans. on Neural Networks, vol. 22, no. 3, pp. 337-346, March 2011.

[19] R. Ak, Y. F. Li, V. Vitelli, E. Zio, E. López Droguett, and C. Magno Couto Jacinto, "NSGA-II-trained neural network approach to the estimation of prediction intervals of scale deposition rate in oil \& gas equipment," Expert Systems with Applications, vol. 40, no. 4, pp. 12051212, March 2013.

[20] A. Konak, D.W. Coit, and A.E. Smith, "Multi-objective optimization using genetic algorithms: A tutorial," Reliability Engineering \& System Safety, vol. 91, no. 9, pp. 992-1007, Sep. 2006.

[21] R., Billinton and R. N. Allan, Reliability Assessment of Large Electric Power Systems. Springer, 1988.

[22] Power Systems Engineering Committee, "Reliability indices for use in bulk power supply adequacy evaluation," IEEE Transactions on Power Apparatus and Systems, vol. PAS-97, no. 4, pp. 1097-1103, July/Aug. 1978.

[23] Moore, R. E., Kearfott, R. B., and Cloud, M. J. Introduction to Interval Analysis, Society for Industrial and Applied Mathematics. 1st ed., USA, 2009, pp. 1-235.

[24] Ross, S. M., 2010. Introduction to Probability Models. $10^{\text {th }}$ Ed., Elsevier Inc., USA.

[25] R. Billinton, S. Kumar, N. Chowdhury, K. Chu, K. Debnath, L. Goel, E. Khan, P. Kos, G. Nourbakhsh,, J. Oteng-Adjei, "A reliability test system for educational purposes-basic data," IEEE Trans. on Power Systems, vol. 4, no. 3, pp. 1238-1244, Aug. 1989.

[26] G. E. P. Box, G. M. Jenkins, and G. C. Reinsel, Time Series Analysis: Forecasting and Control. $4^{\text {th }}$ ed., Wiley, 2008.

[27] Devore, J. L. and Berk, K. N., 2011. Modern mathematical statistics with applications. Springer, London.

[28] Mosteller, F. and Tukey, J. W., 1977. Data Analysis and Regression: A Second Course in Statistics. Addison-Wesley Series in Behavioral Science: Quantitative Methods, Reading, Mass.: Addison-Wesley, 1977, 1.

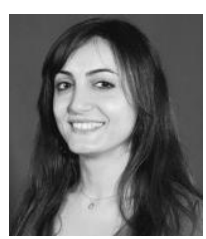

Ronay Ak is currently pursuing the Ph.D. degree at Chair on Systems Science and the Energetic Challenge, European Foundation for New Energy-Électricité de France (EDF), École Centra le Paris (ECP) and École Supérieure d'Électricité (SUPELEC), France, since March 2011. Her research interests include uncertainty quantification, prediction methods, artificial intelligence, reliability analysis of wind-integrated power networks, and multi-objective optimization.

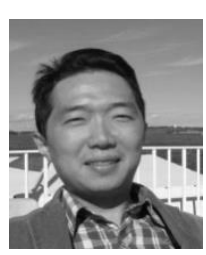

Yan-Fu Li is an Assistant Professor at Ecole Centrale Paris (ECP) \& Ecole Supérieure d'Electricité (SUPELEC), Paris, France. Dr. $\mathrm{Li}$ completed his $\mathrm{PhD}$ research in 2009 at National University of Singapore, and went to the University of Tennessee as a research associate. His current research interests include reliability modeling, uncertainty analysis, evolutionary computing, and Monte Carlo simulation. He is the author of more than 30 publications, all in refereed international journals, conferences, and books. He is an invited reviewer of over 10 international journals.

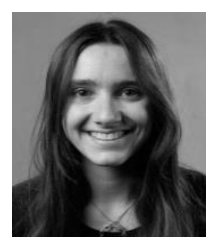

Valeria Vitelli received the Ph.D. degree in Mathematical Models and Methods for Engineering, with a focus on statistical models for classification of high-dimensional data, in May 2012. She worked as a postdoc researcher within the Chair on Systems Science and the Energetic Challenge, European Foundation for New Energy- Électricité de France (EDF), École Centrale Paris (ECP) and École Supérieure d'Électricité (SUPELEC), France, from February 2012 to May 2013. She is currently a postdoc researcher in the Department of Biostatistics, University of Oslo, Norway. Her current research interests concern prediction and uncertainty quantification methods for complex data.

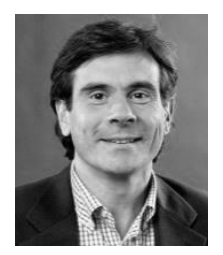

Enrico Zio received the Ph.D. degree in nuclear engineering from Politecnico di Milano and MIT in 1995 and 1998, respectively. He is currently Director of the Chair on Systems Science and the Energetic Challenge, European Foundation for New Energy- Électricité de France (EDF), at École Centrale Paris (ECP) and École Superieure d'Électricité (SUPELEC) and full professor at Politecnico di Milano. His research focuses on the characterization and modeling of the failure/repair/maintenance behavior of components, complex systems and their reliability, maintainability, prognostics, safety, vulnerability and security, Monte Carlo simulation methods, soft computing techniques, and optimization heuristics. 